\title{
Unusual stable trapping of the ultrarelativistic electrons in the Van Allen radiation belts
}

\author{
Yuri Y. Shprits ${ }^{1,2,3 \star}$, Dmitriy Subbotin ${ }^{3}$, Alexander Drozdov ${ }^{3,4}$, Maria E. Usanova ${ }^{5}$, Adam Kellerman ${ }^{3}$, \\ Ksenia Orlova ${ }^{3,4,6}$, Daniel N. Baker ${ }^{7}$, Drew L. Turner ${ }^{3}$ and Kyung-Chan Kim ${ }^{8}$
}

\begin{abstract}
Radiation in space was the first discovery of the space age. Earth's radiation belts consist of energetic particles that are trapped by the geomagnetic field and encircle the planet'. The electron radiation belts usually form a two-zone structure with a stable inner zone and a highly variable outer zone, which forms and disappears owing to waveparticle interactions on the timescale of a day, and is strongly influenced by the very-low-frequency plasma waves. Recent observations revealed a third radiation zone at ultrarelativistic energies $^{2}$, with the additional medium narrow belt (longlived ring) persisting for approximately 4 weeks. This new ring resulted from a combination of electron losses to the interplanetary medium and scattering by electromagnetic ion cyclotron waves to the Earth's atmosphere. Here we show that ultrarelativistic electrons can stay trapped in the outer zone and remain unaffected by the very-low-frequency plasma waves for a very long time owing to a lack of scattering into the atmosphere. The absence of scattering is explained as a result of ultrarelativistic particles being too energetic to resonantly interact with waves at low latitudes. This study shows that a different set of physical processes determines the evolution of ultrarelativistic electrons.
\end{abstract}

Over half a century ago, on the basis of the observations of the first US satellite mission Explorer 1, James Van Allen and colleagues from the University of Iowa discovered the inner radiation belt ${ }^{3}$. The inner belt is very stable and consists of electrons and protons trapped between 1.2 and 2.0 Earth radii. Later, USSR and US missions showed that the radiation belts exhibited a two-zone structure ${ }^{1,4}$; there is an additional outer belt present at higher distances $\left(\gtrsim 3 R_{E}\right)$. A region of relatively low electron fluxes separating the belts is usually referred to as the slot region. The outer belt consists of energetic electrons and is highly dynamic and variable. It is produced by the continuous acceleration of electrons during inward transport (second-order Fermi acceleration and betatron acceleration $)^{5-7}$ and local acceleration due to resonance with plasma waves ${ }^{8-10}$. The energetic electrons are continuously lost to the atmosphere and also regularly depleted as a result of losses to the magnetopause (the boundary of the Earth's magnetosphere $)^{11-13}$.

The two-zone structure has been observed to be altered owing to very unusual acceleration events. Observation of the so-called Halloween superstorm showed that electrons can be accelerated to relativistic energies in the slot region between the two belts. The extreme filling of the slot region in October-November 2003 was produced as a result of local acceleration by very-low-frequency (VLF) plasma waves ${ }^{8,9}$. Another unusual event was observed by the Combined Release and Radiation Effects Satellite on 24 March 1991. A powerful injection of protons and electrons deep into the inner zone was a result of an impulsive acceleration brought on by an interplanetary shock ${ }^{14}$. Recent observations of the ultrarelativistic electrons by NASA's Radiation Belt Storm Probes, renamed by NASA as Van Allen Probes on 9 November 2012 (ref. 15), revealed one of the most surprising configurations of the corpuscular radiation in space seen so far. The newly launched spacecraft observed three radiation zones and two slot regions between them at energies above a few megaelectronvolts (ref. 2). The middle radiation zone (a narrow ring; fraction of the Earth's radius wide) was very stable, lasting for approximately 4 weeks. In this study, we model this unusual event by accounting for diffusive wave-particle interactions and using observations from the geosynchronous (GOES) satellites for the boundary conditions.

Diffusive simulations (Fig. 1b,d,f) driven by the naturally occurring plasma waves ${ }^{16}$ reproduce the formation of the storage ring at ultrarelativistic energies $(>2 \mathrm{MeV})$ shown in Fig. 1c,e. As discussed in more detail below, diffusive local acceleration, which is very efficient at energies below $2 \mathrm{MeV}$ (refs 8,9), becomes inefficient for such high-energy electrons. Unlike during the previous unusual events (that is, the Halloween and 24 March 1991 storms), the new radiation belt that formed on 3 September 2012 was not produced by acceleration, because no additional sources were added to simulations, but rather results from loss of particles above the plasmapause, which moves close to the inner boundary of the outer belt.

At radial distances above $4 R_{E}$, the total electron loss can be a combination of the loss to the interplanetary medium, outward transport of particles and loss to the atmosphere, whereas at lower radial distances rapid loss is produced by scattering into the atmosphere by electromagnetic ion cyclotron (EMIC) waves. Ground and in situ observations show that strong EMIC waves were present during the ring's formation period (Fig. 2a,b). Simulations reproduce the difference in the behaviour between relativistic $(\leqslant 2 \mathrm{MeV})$ and ultrarelativistic $(>2 \mathrm{MeV})$ electrons. The acceleration of relativistic electrons after the storm occurs at a broad range of radial distances owing to inward radial diffusion and local acceleration by VLF waves. In contrast, the ultrarelativistic electrons form a narrow ring that persists for a long time until the arrival of the next large storm on 30 September. The difference in behaviour of the relativistic and ultrarelativistic particles is due

\footnotetext{
${ }^{1}$ Skolkovo Institute of Science and Technology, Skolkovo 143025, Russia, ${ }^{2}$ Department of Earth and Space Sciences, Massachusetts Institute of Technology, Cambridge, Massachusetts 02139, USA, ${ }^{3}$ University of California Los Angeles, Los Angeles, California 90095, USA, ${ }^{4}$ Lomonosov Moscow State University Skobeltsyn Institute of Nuclear Physics, Moscow 119991, Russia, ${ }^{5}$ Department of Physics, University of Alberta, Edmonton, Alberta P6G 2M7, Canada, ${ }^{6}$ University Corporation for Atmospheric Research, Boulder, Colorado 80301, USA, ${ }^{7}$ LASP, University of Colorado, Boulder, Colorado 80303-7814, USA,

${ }^{8}$ Korea Astronomy and Space Science Institute, Daejeon 305-348, Korea. *e-mail: shprits@mit.edu
} 
a

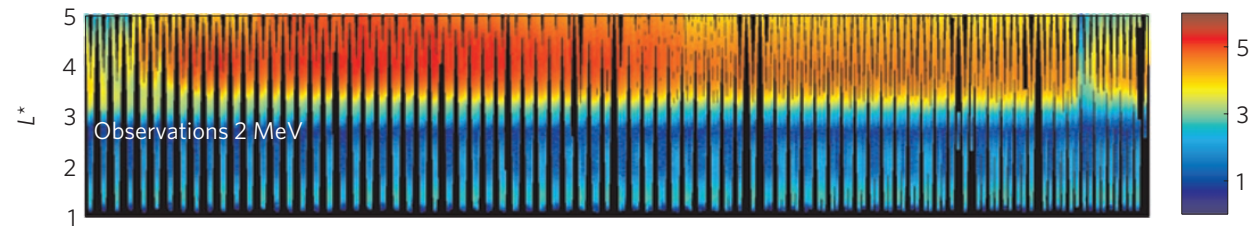

b
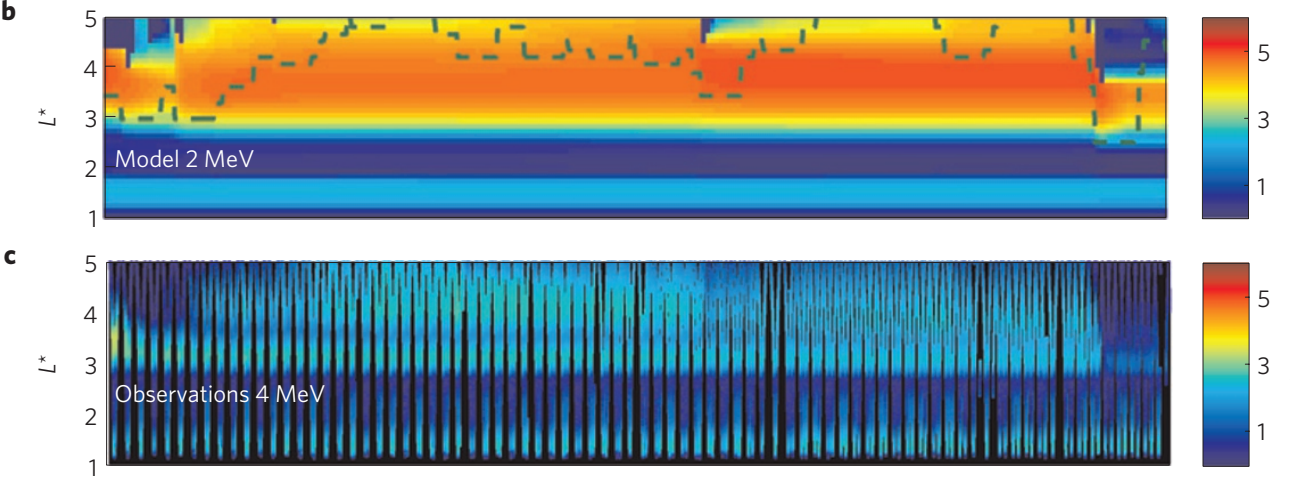

d

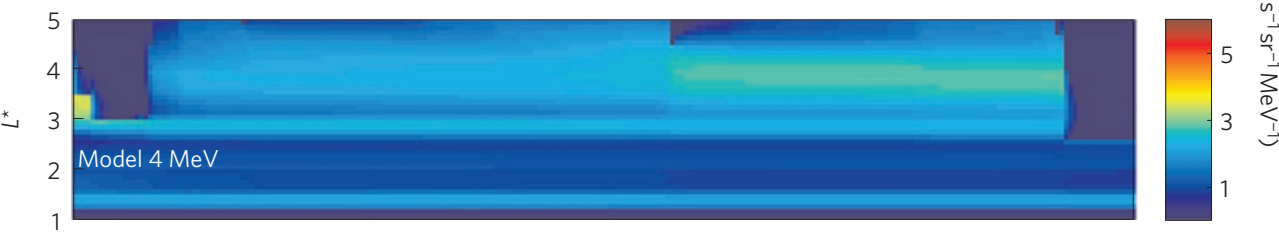

e
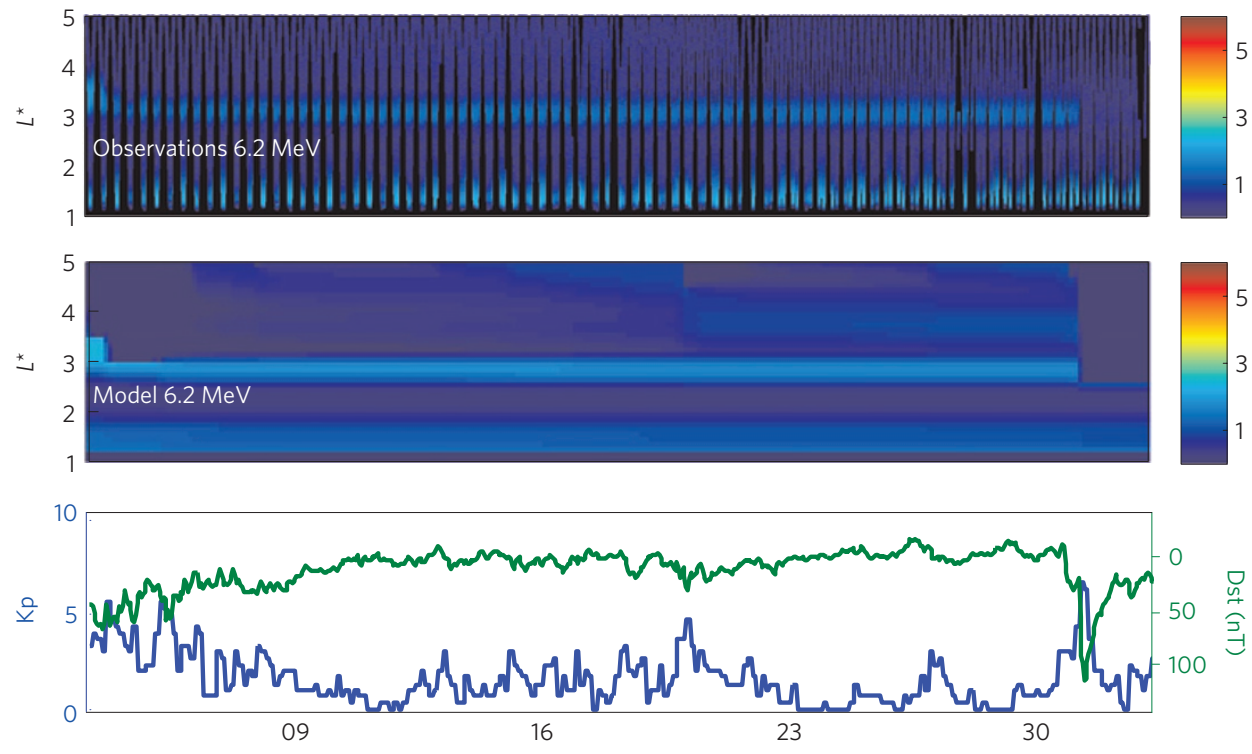

Day of September 2012

Figure 1 | Comparison of diffusive transport simulations with observations. $\mathbf{a}$, Observation from the Van Allen Probes of electrons with a kinetic energy of $2 \mathrm{MeV}$. b, Diffusive simulations at $2 \mathrm{MeV}$. c,d, The same as in $\mathbf{a}, \mathbf{b}$ but for $4 \mathrm{MeV}$ electrons. e,f, The same as in a,b but for $6.2 \mathrm{MeV}$ electrons. Simulations account for radial diffusion of electrons, diffusive loss to the atmosphere, and local acceleration due to quasi-linear diffusive interactions with various plasma waves inside and outside the plasmasphere (the area of cold and dense plasma of ionospheric origin co-rotating with the Earth). The plasmapause (the upper boundary of the plasmasphere) is shown as a green line and is modelled according to ref. 18 on $\mathbf{b}$. a-f show omni-directional electron fluxes as a function of time and $L^{*}$. The $L^{*}$ or $L$-shell parameter is effectively the radial distance from the centre of the Earth to the magnetic field line in the equatorial plane measured in Earth radii. Simulations reproduce the unusual dynamics of the radiation belts and the formation of the long-lived storage ring at ultrarelativistic energies. The storage ring formed near $L^{*}=3$ on 3 September 2012 for $E>2 \mathrm{MeV}$ and persisted until the arrival of a coronal mass ejection that induced another storm on 30 September 2012. The bottom panels show indices of geomagnetic activity Kp and Dst derived from the fluctuations of the magnetic field measured on the ground. High Kp and low Dst values correspond to elevated levels of geomagnetic activity.

to the difference in wave-particle interactions between different energy particles and plasma waves.

After the flux dropout on 3-5 September, the electrons in the long-lived ring found themselves inside the plasmasphere. Whereas relativistic electrons can be scattered by hiss plasma waves inside the plasmasphere and are usually lost on a scale of a few days (Fig. 2c, blue lines), ultrarelativistic electrons of the long-lived storage ring turn out to be too energetic to be effectively scattered by hiss waves 


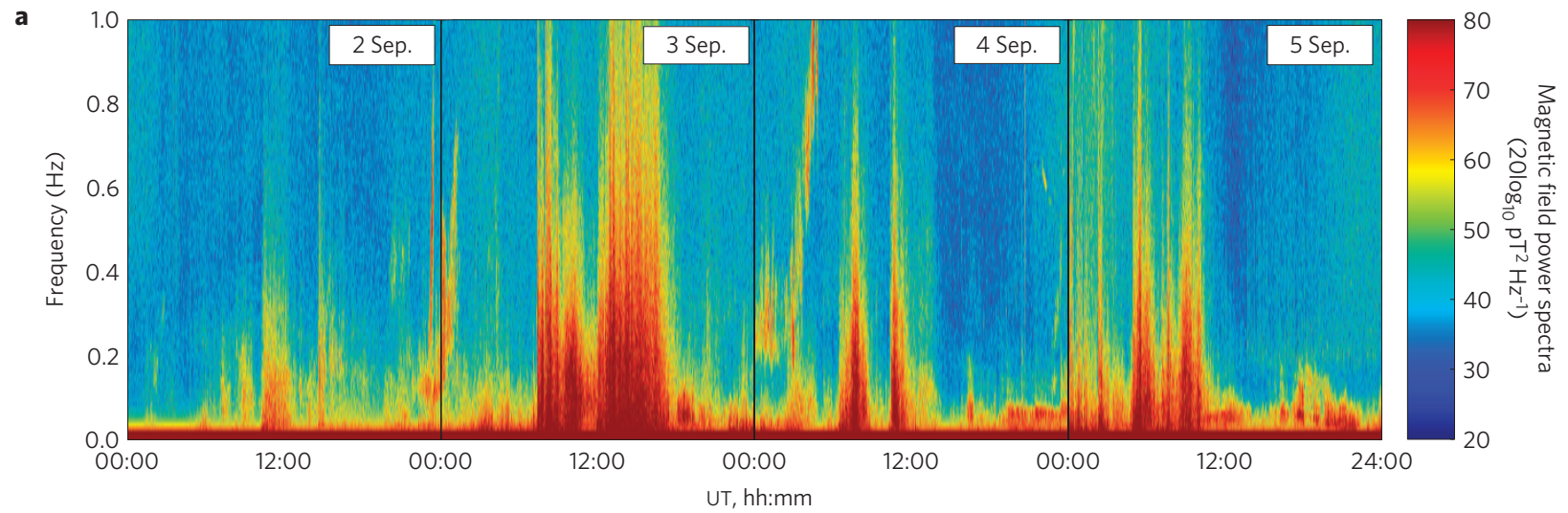

b

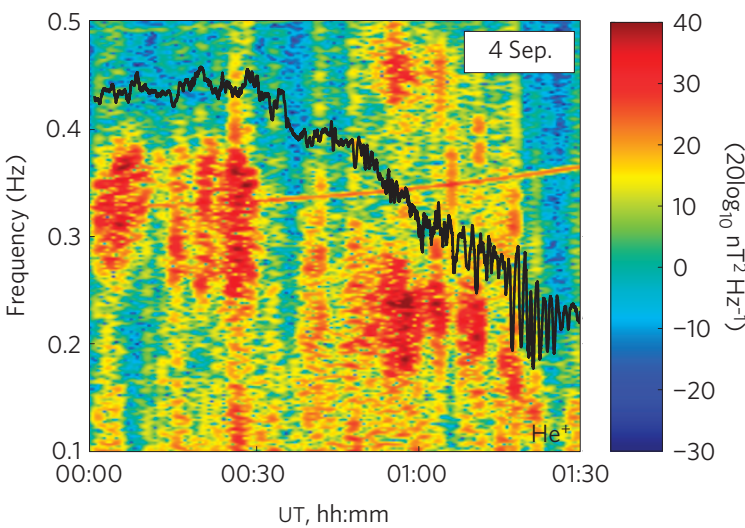

c

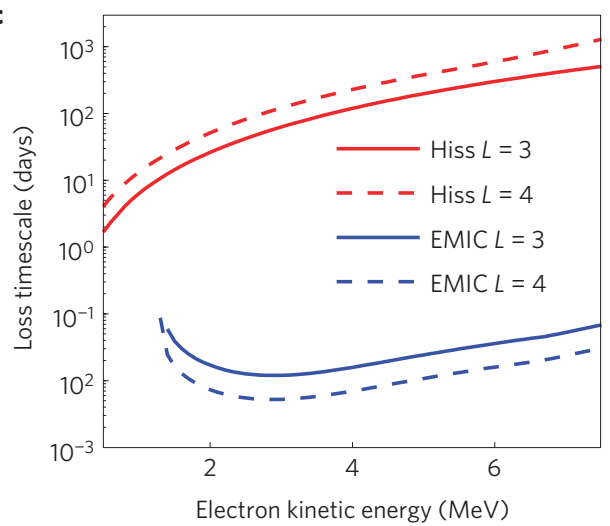

Figure 2 | Wave observations and estimated timescales. a, Spectrograms of the magnetic field measured by The Canadian Array for Real-time Investigations of Magnetic Activity (CARISMA; www.carisma.ca) ground-based magnetometer ${ }^{19}$ between 3 and 5 September. The waves measured on the ground correspond to radial distances of $L \sim 4 \pm 1 R_{E}$. b. Dynamic spectrogram of in situ magnetic field clearly shows EMIC waves below the He gyrofrequency (black line). These measurements are used to specify the power spectral density of EMIC waves for the loss calculations. c, Calculated electron loss timescales at different radial distances ( $L=3$ and 4$)$ as a function of electron kinetic energy. Loss timescales are estimated as an inverse quasi-linear diffusion rate of particles for which the mirror points of their bounce trajectories are close to the atmosphere. EMIC waves provide the dominant and very fast scattering of electrons during the storms (blue line) and scatter particles outside the plasmasphere into the atmosphere on the timescales of less than a day ${ }^{13}$. EMIC waves do not affect electrons at lower energies. Hiss emissions inside the plasmasphere can provide loss on timescales comparable to a day at megaelectronvolt energies, but the loss timescale increases with energy up to a thousand days. Scattering rates are computed for quiet geomagnetic conditions.

inside the plasmasphere, and decay on a scale of up to a thousand days, as shown in Fig. 2c, red line.

Particles are most efficiently scattered when the Doppler-shifted wave frequency is equal to integer multiples of the particle's relativistic gyrofrequency (that is, gyroresonance). Figure 3 illustrates the first-order resonance condition by presenting areas of Doppler-shifted wave frequency and relativistic gyrofrequency. Relativistic particles at energies of $1 \mathrm{MeV}$ can be efficiently scattered by waves (Fig. 3a,d), which explains the fast acceleration by chorus waves outside the plasmasphere and fast loss timescales for these particles by hiss waves inside the plasmasphere (Fig. 1a). Ultrarelativistic particles produce more Doppler shifting, and ultrarelativistic electrons near the equatorial plane find themselves out of dominant first-order resonance with waves that are present inside the plasmasphere (Fig. 1c,e). Figure $3 c, f$ demonstrates that ultrarelativistic particles outside or inside the plasmasphere may be in resonance with whistler mode hiss or chorus waves only at high latitudes, where the magnetic field is very strong. When the magnetic field is strong, scattering of particles by waves becomes less efficient, which results in the low scattering rates at high latitudes.

During the storm, electrons with energies from $\sim 100 \mathrm{keV}$ to $\sim 2 \mathrm{MeV}$ can be accelerated locally by chorus waves over a broad range of $L$-shells, which explains the formation of the wide outer belt at relativistic energies. Ultrarelativistic electrons with energies above $2 \mathrm{MeV}$ will not be in resonance with chorus waves near the equator and are not diffused in energy by chorus waves, resulting in the formation of the narrow ring observed at $L \sim 3.3$ (Fig. 3b,c).

After the storm electrons at low $L$-shells find themselves inside the plasmasphere in a very different plasma environment, where EMIC waves are not present and the strongest emissions are hiss whistler mode plasma waves. Energetic and relativistic electrons will resonate with hiss waves near the equator (Fig. 3d) and will be lost to the atmosphere on a timescale of a few days. Ultrarelativistic electrons will be out of resonance with hiss waves near the equator (Fig. 3e,f), which is the reason why the unusual storage ring can persist for over four weeks before being wiped out by the arrival of the next strong storm.

The observations of the unusual ring ${ }^{2}$ together with the analysis presented in this manuscript completely change the way we think about the radiation belts. The radiation belt community has been discussing radial diffusion versus local acceleration by VLF waves and scattering into the atmosphere by VLF (chorus and hiss plasma waves) since the early years of the space age and in much more detail during the recent decade $8,13,17$. Our study definitively shows 


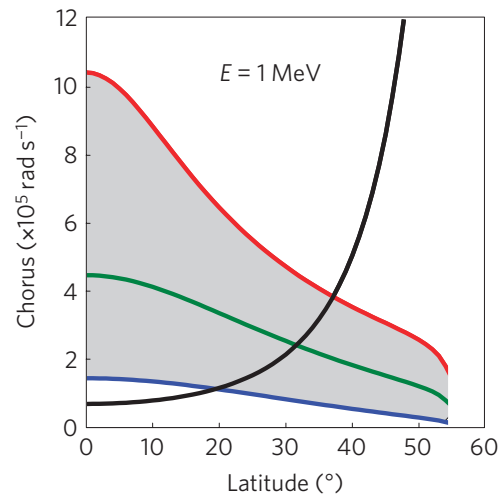

d

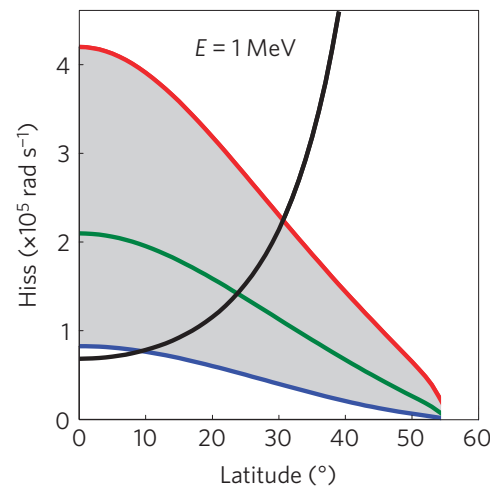

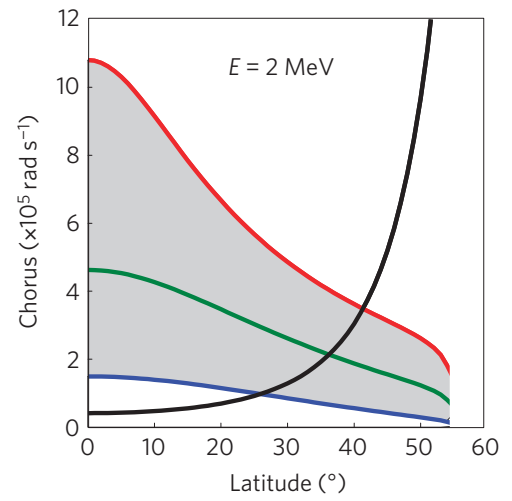

e

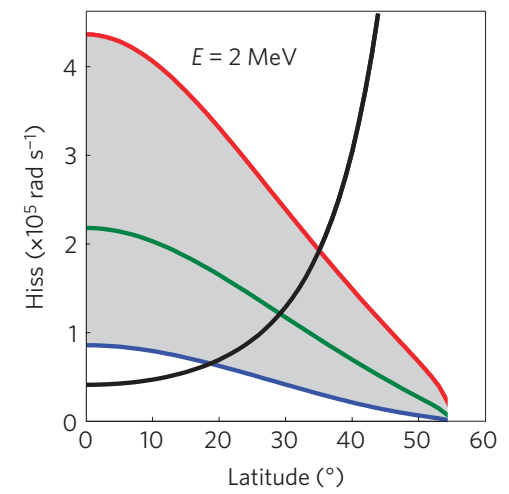

C

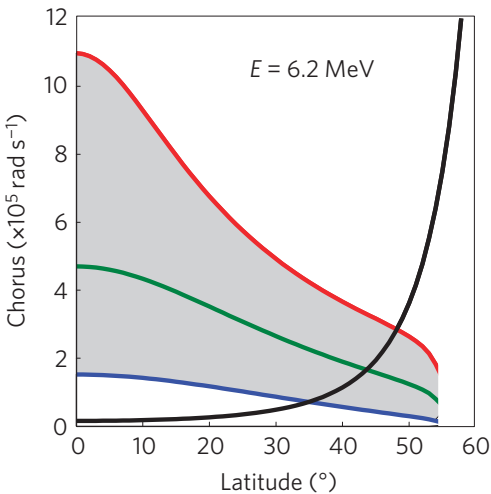

f

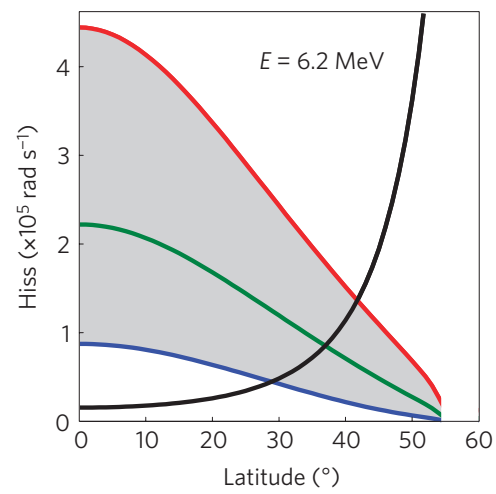

Figure 3 | First-order resonance condition. a-f, Illustration of the resonance conditions for relativistic and ultrarelativistic electrons interacting with chorus waves outside the plasmasphere (a-c) and hiss waves inside the plasmasphere (d-f). The blue (red) lines show the Doppler-shifted lower (upper) bound frequency for hiss and chorus waves. The green lines indicate the frequency of maximum wave power. The black lines represent the relativistic electron

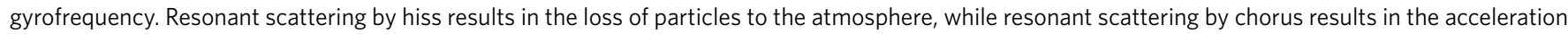
of particles. The resonance condition is satisfied when the Doppler-shifted wave frequency equals the relativistic gyrofrequency. The intersection of the grey area and the black line shows possible resonance latitudes. $6.2 \mathrm{MeV}$ electrons can resonate only at high magnetic latitudes ( $>30^{\circ}$ ) where wave-particle interactions become inefficient owing to the strong background magnetic field.

that at electron energies above approximately $2 \mathrm{MeV}$ a different set of physical processes determines the evolution of the radiation belts. In many ways the situation is much simpler in the case of ultrarelativistic electrons, as considered in this study. Diffusive interactions with VLF waves including chorus and hiss do not play a significant role as they are out of first-order resonance with ultrarelativistic particles near the geomagnetic equator. This simplicity in a sense is responsible for the formation of a very unusual narrow ring and also explains why the particles can stay in the ring for so long. This study shows that we cannot consider the electron radiation belts as a trapped bulk population at an energy greater than approximately $1 \mathrm{MeV}$. Physical processes responsible for acceleration and loss of electrons at ultrarelativistic energies are different and fluxes can exhibit very different spatial structures as a function of energy.

Received 27 March 2013; accepted 15 August 2013; published online 22 September 2013

\section{References}

1. Van Allen, J. A. Discovery of the Magnetosphere Vol. 7, 235-251 (History of Geophysics, American Geophysical Union, 1997).

2. Baker, D. N. et al. A long-lived relativistic electron storage ring embedded in earth's outer Van Allen Belt. Science 340, 186-190 (2013).

3. Van Allen, J. A. \& Frank, L. A. Radiation around the Earth to a radial distance of 107,400 km. Nature 183, 430-434 (1959).

4. Panasyuk, M. I. Coming of age and development of space physics at Moscow State University. Radiation in space: The legacy of S. N. Vernov. Phys. Usp. 54, 190-202 (2011).
5. Hudson, M. K., Elkington, S. R., Lyon, J. G. \& Goodrich, C. C. Increase in relativistic electron flux in the inner magnetosphere: ULF wave mode structure. Adv. Space Res. 25, 2327-2337 (2000).

6. Schulz, M. \& Lanzerotti, L. J. Particle Diffusion in the Radiation Belts (Springer, 1974).

7. Lanzerotti, L. J., Maclenna, C. G. \& Schultz, M. Radial diffusion of outer-zone electrons-an empirical approach to third-invariant violation. J. Geophys. Res. 75, 5351-5371 (1970).

8. Horne, R. B. et al. Wave acceleration of electrons in the Van Allen radiation belts. Nature 437, 227-230 (2005).

9. Baker, D. N. et al. An extreme distortion of the Van Allen belt arising from the Halloween solar storm in 2003. Nature 878-881 (2004).

10. Shprits, Y. Y. et al. Acceleration mechanism responsible for the formation of the new radiation belt during the 2003 Halloween solar storm. Geophys. Res. Lett. 33, L05104 (2006).

11. Shprits, Y. Y. et al. Outward radial diffusion driven by losses at magnetopause. J. Geophys. Res. 111, A11214 (2006).

12. Turner, D. L., Shprits, Y., Hartinger, M. \& Angelopoulos, V. Explaining sudden losses of outer radiation belt electrons during geomagnetic storms. Nature Phys. 8, 208-212 (2012).

13. Millan, R. M. \& Thorne, R. M. Review of radiation belt relativistic electron losses. J. Atmos. Solar Terr. Phys. 69, 362-377 (2007).

14. Blake, J. B., Kolasinski, W. A., Fillius, R. W. \& Mullen, E. G. Injection of electrons and protons with energies of tens of $\mathrm{MeV}$ into $L<3$ on 24 March 1991. Geophys. Res. Lett. 19, 821-824 (1992).

15. Mauk, B. H. et al. Science objectives and rationale for the radiation belt storm probes mission. Space Sci. Rev. 1-25 (2012).

16. Shprits, Y.Y., Subbotin, D. \& Ni, B. Evolution of electron fluxes in the outer radiation belt computed with the VERB code. J. Geophys. Res. 114, A11209 (2009).

17. Shprits, Y. Y., Elkington, S. R., Meredith, N. P. \& Subbotin, D. A. Review of modeling of losses and sources of relativistic electrons in the outer radiation belts: I. Radial transport. J. Atmos. Sol. Terr. Phys. 70, 1679-1693 (2008). 
18. Carpenter, D. L. \& Anderson, R. R. An ISEE/whistler model of equatorial electron density in the magnetosphere. J. Geophys. Res. 97, 1097-1108 (1992).

19. Mann, I. R. et al. The upgraded CARISMA magnetometer array in the THEMIS era. Space Sci. Rev. 141, 413-451 (2008).

\section{Acknowledgements}

This research was supported by a Presidential Early Career Award for Scientists and Engineers (PECASE) award grant to Y.Y.S. This research was also supported by the UC-Lab Fee programme, NASA grants NNX10AK99G, NNX09AF51G and

NNX13AE34G and NSF AGS-1203747. We thank the Van Allen Probes team. ECT funding was provided by the Johns Hopkins University Applied Physics Laboratory (JHU/APL) contract no. 976399. We thank I. R. Mann, D. K. Milling and the rest of the CARISMA team. CARISMA is operated by the University of Alberta, funded by the Canadian Space Agency(CSA). K.O. was supported by the NASA LWS Jack Eddy Postdoctoral Fellowship Program, administrated by UCAR. D.L.T. is grateful for support from NASA THEMIS contract NAS5-02099 and grant NNX12AJ55G and the
MAARBLE project. M.E.U. was supported by the Canadian Space Agency and the MAARBLE FP7 project.

\section{Author contributions}

Y.Y.S. initiated the study and led modelling, analysis of observations, and writing of the manuscript. D.S. modelling, A.D. analysis of observations, M.E.U. analysis of observations, A.K. modelling and analysis of observations, K.O. modelling, D.N.B. provided Van Allen Probes data, D.L.T. provided THEMIS data, K-C.K. modelling.

\section{Additional information}

Reprints and permissions information is available online at www.nature.com/reprints. Correspondence and requests for materials should be addressed to Y.Y.S.

\section{Competing financial interests}

The authors declare no competing financial interests. 